\title{
Research regarding reverse engineering for aircraft components
}

\author{
Razvan Udroiu' ${ }^{1, *}$ \\ ${ }^{1}$ Transilvania University of Brasov, Department of Manufacturing Engineering, Mihai Viteazu No.5, \\ Brasov, Romania
}

\begin{abstract}
Reverse engineering is a useful technique used in manufacturing and design process of new components. In aerospace industry new components can be developed, based on existing components without technical Computer Aided Design (CAD) data, in order to reduce the development cycle of new products. This paper proposes a methodology wherein the CAD model of turbine blade can be build using computer aided reverse engineering technique utilising a 5 axis Coordinate Measuring Machine (CMM). The proposed methodology uses a scanning strategy by features, followed by a design methodology for 3D modelling of complex shapes.
\end{abstract}

\section{Introduction}

In present, most companies face a huge demand for diversified products from customers. They must adapt to the different requirements, through experimentation methods, techniques, and innovative actions on the market.

Traditionally a technical drawing or Computer Aided Design (CAD) model is created before the manufacturing of the part. But in several cases, where the CAD model does not exist, or the design of the product should be amended because of poor performance or manufacturing difficulties, Reverse Engineering (RE) technology becomes indispensable. The purpose of reverse engineering technology [1-3] is to capture the geometry of an existing physical model, through digitization, and to create a 3D virtual model for use in $\mathrm{CAD}$, Computer Aided Engineering (CAE), Computational Fluid Dynamics (CFD) or Computer Aided Manufacturing (CAM) software. Liang \& Grier [4] have defined the reverse engineering as a process of obtaining a geometric CAD model from 3D measurements acquired by contact or non-contact scanning technique of an existing physical model. The purpose of RE results from the following reasons:

- Manufacturing of products for which there is not a 3D CAD model,

- Optimize the shape of the products (redesign) for which 3D date is not available [5],

- Development of new products based on existing one with no CAD data,

- Development of customized products (medical prostheses and sports equipment),

- Inspect object dimension, deformations, etc. to confirm the quality and performance by computer-aided engineering analysis and inspection,

*Corresponding author: udroiu.r@unitbv.ro 
- The repair and maintenance of complex products [6].

The main methods of capturing 3D geometry information on a product are classified into contact methods and non-contact methods. Which method is most attractive in terms better, faster, cheaper? Fast scanners will not be as accurate, more accurate scanners will cost more money. This means that you will need to choose up to two out of the three available factors: accuracy, speed or cost [7]. Computer Aided Inspection (CAI) and Computer Aided Reverse Engineering (CARE) are two techniques which use contact or non-contact methods and computer aided tools.

Contact methods comes in two main varieties: stationary CMMs and portable CMMs. Stationary coordinate measuring machines (CMMs) are large installation/ systems that are highly accurate, expensive and much slower compared to non-contact methods [7].

The noncontact solutions are a relatively new approach which consists in laser scanners, structured light scanners and industrial CT scanners (computed tomography). The advantages of non contact scanners over contact devices include fast data collection, and more comprehensive collection of points means a clearer view of the entire part. There are many parameters that affect the optical methods and hence the quality of scanning data, such as: reflective surfaces, narrow openings and sharp edges that can be difficult to scan. Thus, translucent, reflective or black / near-black parts require preprocessing operations, consisting in spraying with matte white powder of the surface, which affect the accuracy of the measurement in a range between 0.003-0.005 $\mathrm{mm}$ [8].

This paper proposes a 3D contact scanning methodology and a design reconstruction of a component, with applications for turbine blades. The case study regarding the 3D scanning of a turbine blade from Tumansky R-13 turbojet engine is a result of research from Aircraft engineering specialization from Transilvania University of Brasov. The Tumansky R-13 is a turbojet engine used by some variants of MiG-21 aircraft and Sukhoi Su-15M.

\section{3D scanning of a turbine blade using CMM machine}

The modern aerospace industry uses reverse engineering method as a key to the future of aerospace manufacturing as CAD tool [8-10]. Following the main purpose of RE, capturing and converting a discrete data set into a $3 \mathrm{D}$ model, one of the most used scanning techniques is contact scanning using a coordinate measuring machine (CMM).

\subsection{Preparing the data for CMM scanning}

The main steps before starting the scanning process of the blade consist in the following operations: define the scanning tools, tools calibration, define the device used for positioning of the blade on the CMM table and the alignment of the device.

The turbine blade was 3D scanning using a DEA Global Brown \& Sharpe 5 axis CMM machine having a Renishaw SP $600 \mathrm{M}$ scanning probe with stylus tools attached on it (Fig.1.a.). The stylus is the first link between CMM and workpiece its choice being determined by the geometry of the workpiece [11].

Two stylus tip type was chosen, the first one a ball tip, used for scanning the surface of the blade, and the other a cylinder tip, used for scanning the sharp edges of the blade. The ball tip stylus is the most frequently used type of stylus, suitable for the majority of applications. The ball tip stylus tool has the following main characteristics: $8 \mathrm{~mm}$ diameter ruby ball and $100 \mathrm{~mm}$ in length of a ceramic stem. The cylinder stylus is used for thin workpieces with which proper contact cannot be guaranteed with ball tip stylus. An $8 \mathrm{~mm}$ diameter ruby cylinder stylus was chosen in order to scanning the sharp edges of the turbine blade. Renishaw's SP600 M analogue scanning probes having a high speed scanning up to 
$300 \mathrm{~mm} / \mathrm{s}$ and high frequency response offers high-performance digitising and profile scanning capability [11].

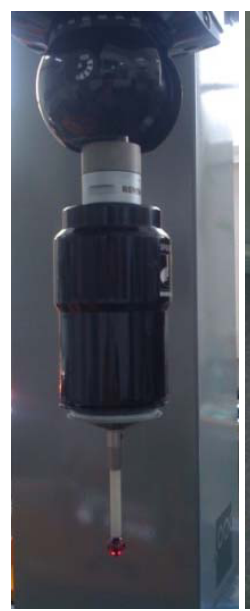

a)

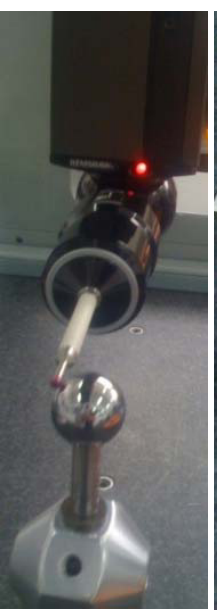

b)

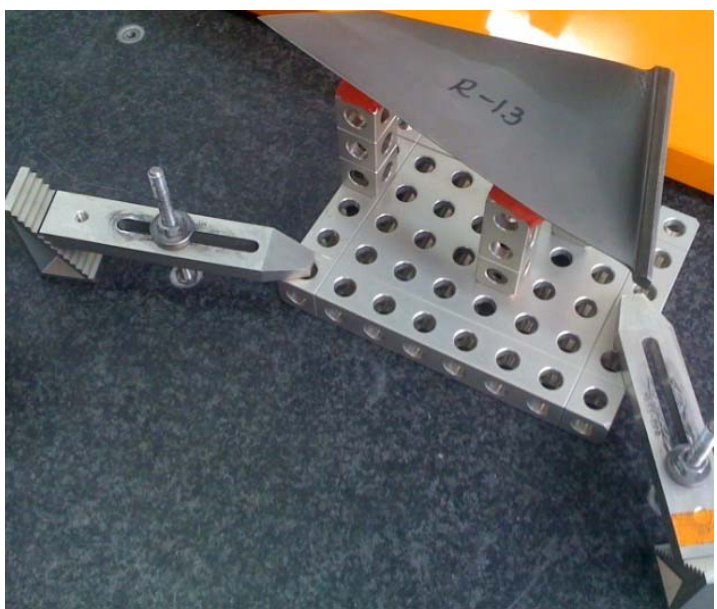

c)

Fig. 1. a) Renishaw SP $600 \mathrm{M}$ scanning probe, b) calibration procedure and c) the device used for positioning the turbine blade.

The probe calibration procedure (Fig.1.b.) consists in measuring of minimum 25 points on a ceramic sphere having $20.002 \mathrm{~mm}$ in diameter, establishing the stylus tip's effective diameters when measuring, and their positions relative to machine's coordinate system. The measurement speed in calibration procedure is recommended to be the same with the scanning speed of the workpiece. The results of the calibration procedure are saved in PCDMIS software and show a $0.003 \mathrm{~mm}$ standard deviation. PC-DMIS CMM is coordinate measurement machine software used to measure everything from simple prismatic parts to the most complex aerospace and automotive components. The device used for positioning of the blade on the CMM table is shown in the Fig.1.c.

The next preparing step consists in defining of the workpiece coordinate system. The origin of the workpiece coordinate system was chosen in the centre of the hole of the foot blade (Fig.2.).
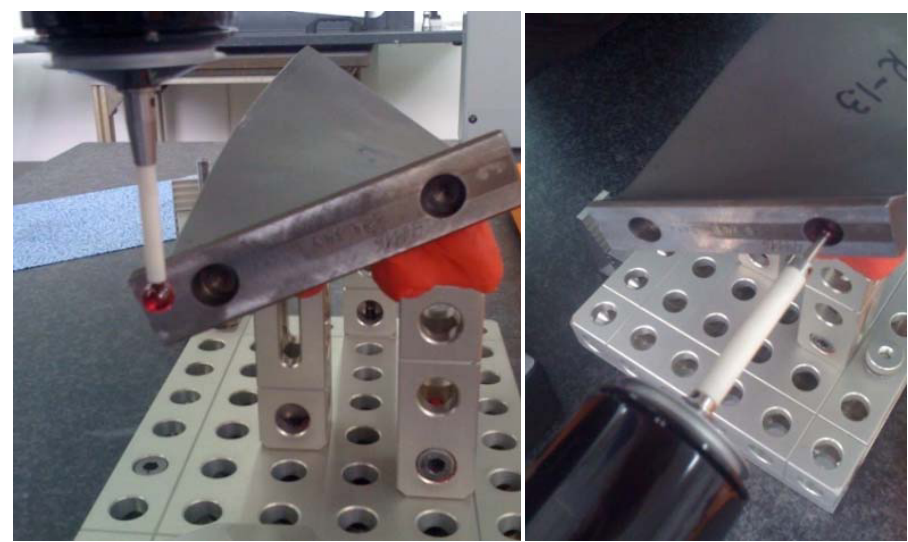

Fig. 2. Defining of the workpiece coordinate system. 


\subsection{The blade scanning using the CMM machine}

Based on the complex geometry of the turbine blade, the geometry of the blade was decomposed in the following main features:

- Blade root feature,

- The main surface feature consisting in upper and lower surfaces of the blade,

- The boundary feature of the blade,

- The link surface feature between the root and the main surface of the blade.

The scanning methodology of the blade was made taking into accord these features.

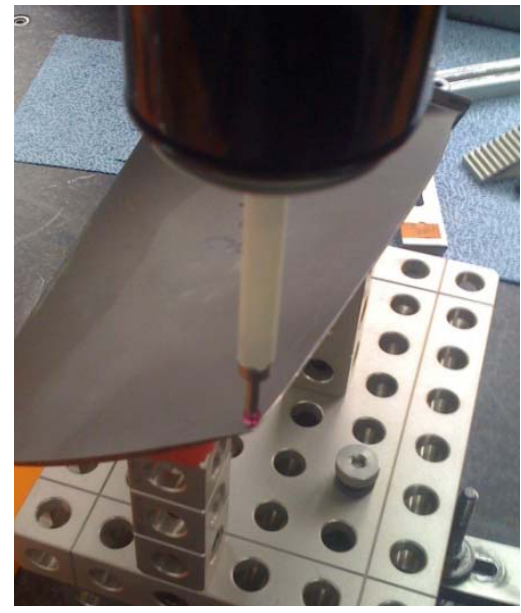

a)

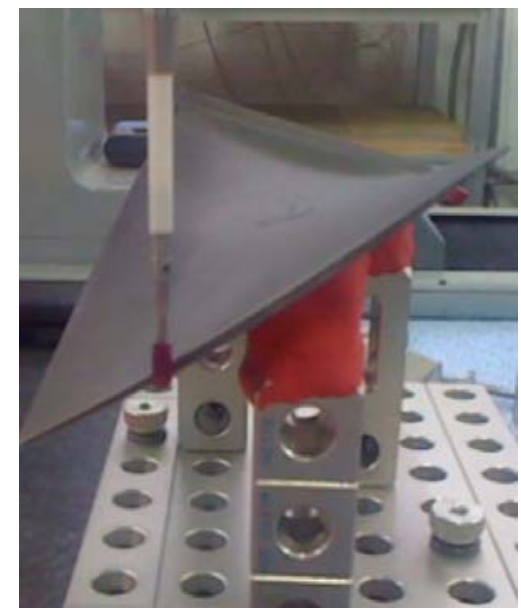

b)

Fig. 3. Scanning process of the blade features for a) the upper and lower surface, b) the sharp boundary feature.

The upper and lower surfaces of the blade and the link surface were scanned using a ball tip stylus, following a patch scan strategy (Fig.3.a.). An open linear strategy was used for scanning the boundary feature of the blade using a cylinder tip stylus (Fig.3.b.). The boundary feature consists of sharp edges. The resulting scanning paths are shown in Fig.4. The programming of the scanning paths was made using PC-DMIS software, based on the blade features. The point cloud data resulting from 3D scanning were exported in IGES file format.

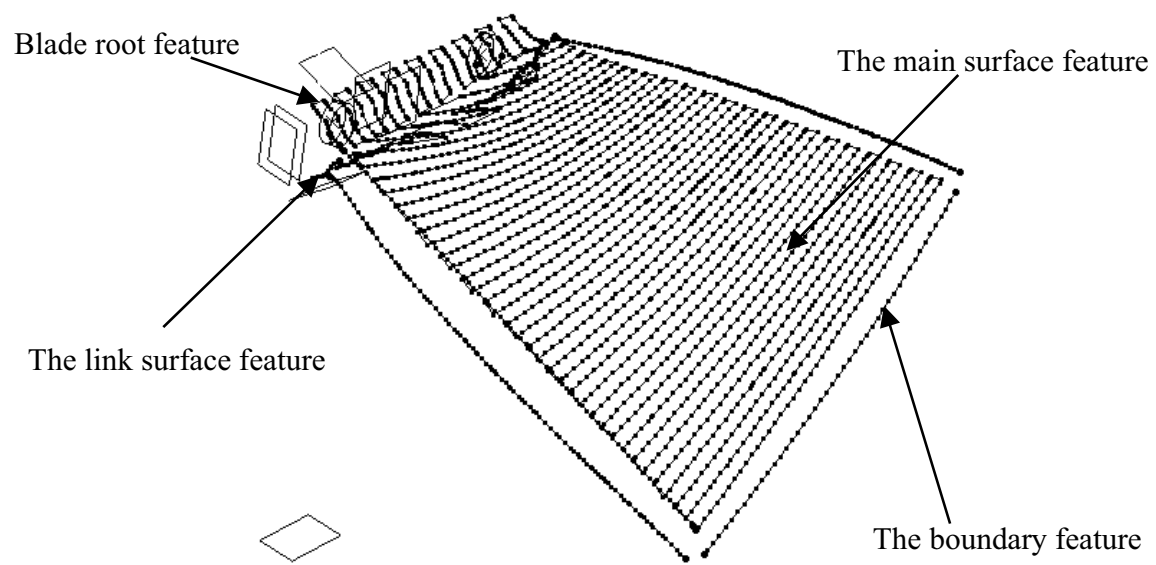

Fig. 4. The scanning paths of the blade features. 


\section{3D modelling of the scanning date using CATIA software}

There are several inspection / reverse engineering software products on the market, bridges the gap between the point collection and the design requirements. Thus can be mentioned standalone software products such as GEOMAGIC, RAPIDFORM, GOM Inspect or some software modules of the complex software systems, e.g. Catia, Creo and SolidWorks.

The design methodology (Fig.5.) proposed for 3D modelling of the turbine blade, is made using Catia version 5 release 21 by Dassault Systemes.

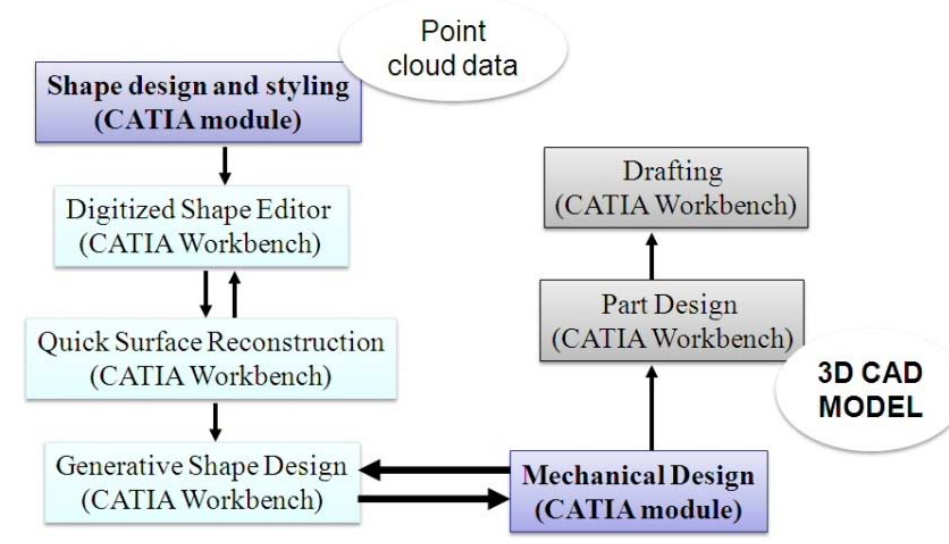

Fig. 5. Design methodology of the blade.

The point cloud data obtained from scanning process of the blade were imported in Digitized Shape Editor workbench of CATIA. Digitized Shape Editor is a CATIA's workbench that allows importing and processing of clouds of points [12]. These clouds of points are used in Quick Surface Reconstruction and then in Generative Shape Design, in order to obtain the blade surface. Following the methodology from Fig.5 was obtained the 3D blade model of the turbine blade (Fig.6.).
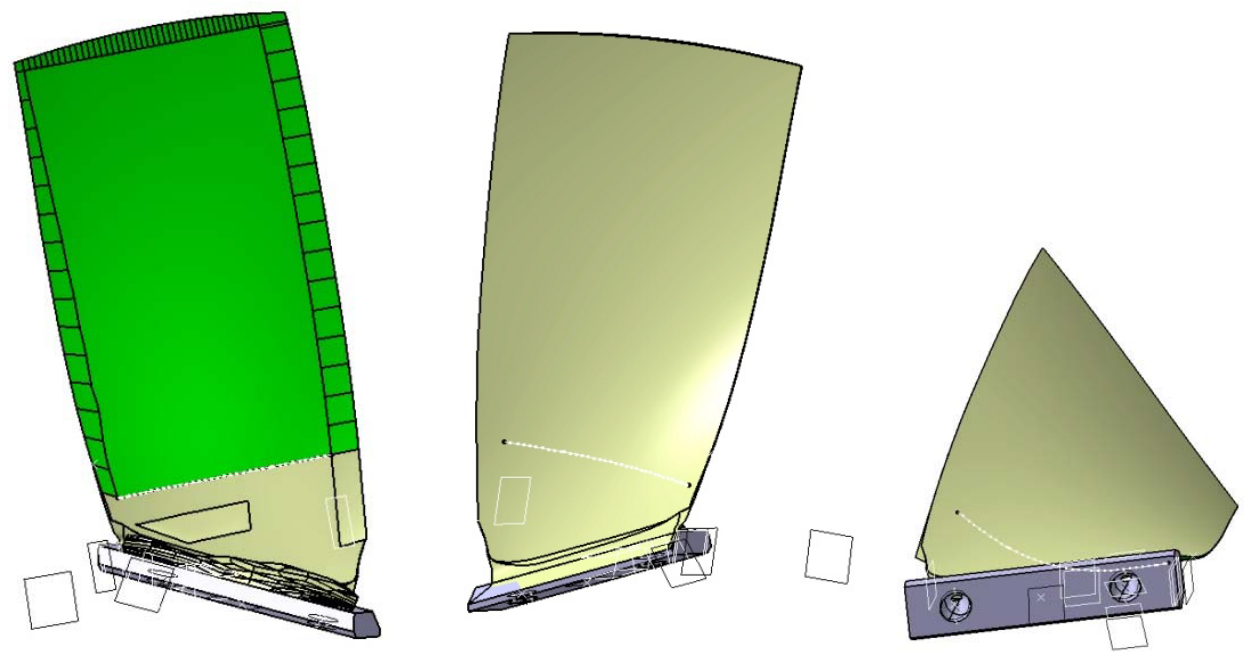

Fig. 6. 3D model of the scanned blade. 
The design methodology of the blade using CATIA consists in the following main steps:

- Import of scanning data (clouds of points),

- Alignment and union of the clouds of points in one cloud of point,

- Processing of cloud of point,

- Generating of curves and surfaces,

- Check the quality of the surfaces,

- Save the 3D model of the blade in IGES or STL file format.

\section{Conclusions}

Reverse engineering is an important method utilized in manufacturing and design process. When there is no technical data of a product or it is unusable, the CAD model is obtained with this method. Computer Aided Reverse Engineering (CARE) integrated into CAD/ $\mathrm{CAE} / \mathrm{CAM}$ systems allows rapid product development by redesigning and optimizing the product, in a short period and decreasing the manufacturing cost.

The turbine blade has a complex shape and required a scanning process by features. The proposed scanning methodology using features, presented in this paper can be used also for other complex parts from aerospace industry.

The errors from scan programming could produce some damage of the stylus, scanning probe or scanning part. The CMM operator must be specialized and he should pay attention to the scanning process. Compared with non-contact scanning, contact scanning presents higher risk factor regarding the influence of the scanning programming relative to the damage of some CMM components, but the scanning accuracy is better.

A future research will be focused on comparing of contact and non-contact scanning procedures of the turbine blade in terms of accuracy, speed and cost.

\section{References}

1. V. Raja, K.J. Fernandes, Reverse Engineering: An Industrial Perspective (SpringerVerlag London Limited, 2008)

2. M. Sokovic, J. Kopac, J. of Mat. Proc. Techn., 175, 398 (2006)

3. R. Vinesh, J.F. Kiran, Reverse Engineering - An Industrial Perspective (Springer, 2008)

4. L.C. Chen, G.C.I Lin, Robot. Comput.-Integr. Manuf., 16,161 (2000)

5. V. Tut, A. Tulcan, C. Cosma, I. Serban, Int. J. of Mech., 4, 79 (2010)

6. D. Tsakatikas, G. Kaisarlis, 4th International Conference on NDT, 4 (2007)

7. ***, Contact vs. Noncontact Measurement for Computer-Aided Inspection, Quality Magazine,http://www.qualitymag.com/articles/91150-contact-vs-noncontactmeasurement-for-computer-aided-inspection (2013)

8. A.A.G. Madrigal, H.R.S. Carrillo, J.O.F. Cavazos, Memorias del XIX Congreso internacional anual de la SOMIM, 897 (2013)

9. K. Mohaghegh, M.H. Sadeghi, A. Abdullah, Int. J. Adv. Manuf. Tech., 32, 1009 (2007)

10. E. Savio, L. De Chiffre, R. Scmitt, Annals of the CIRP, 56, 810 (2007)

11. ***, Renishaw, http://www.renishaw.com/en/products--32083, (2016)

12. ***, CATIA documentation, http://www.3ds.com/support/documentation/overview/ (2015) 